\title{
BMJ Open Impact of a pharmacist-delivered discharge and follow-up intervention for patients with acute coronary syndromes in Qatar: a study protocol for a randomised controlled trial
}

\author{
Amani Zidan, ${ }^{1}$ Ahmed Awaisu, ${ }^{1}$ Nadir Kheir, ${ }^{1}$ Ziyad Mahfoud ${ }^{2}$ Rasha Kaddoura, ${ }^{3}$ \\ Sumaya AlYafei, ${ }^{3}$ Maguy Saffouh El Hajj ${ }^{1}$
}

To cite: Zidan A, Awaisu A, Kheir N, et al. Impact of a pharmacist-delivered discharge and follow-up intervention for patients with acute coronary syndromes in Qatar: a study protocol for a randomised controlled trial. BMJ Open 2016;6:e012141. doi:10.1136/bmjopen-2016012141

- Prepublication history for this paper is available online. To view these files please visit the journal online (http://dx.doi.org/10.1136/ bmjopen-2016-012141).

Received 4 April 2016 Revised 5 October 2016 Accepted 27 October 2016

CrossMark

${ }^{1}$ Clinical Pharmacy and Practice Section, Qatar University College of Pharmacy, Doha, Qatar ${ }^{2}$ Weill Cornell Medicine-Qatar, Doha, Qatar

${ }^{3}$ Heart Hospital, Hamad Medical Corporation, Doha, Qatar

Correspondence to Dr Maguy Saffouh El Hajj; maguyh@qu.edu.qa

\section{ABSTRACT}

Introduction: Acute coronary syndrome (ACS) is one of the leading causes of morbidity and mortality worldwide. Secondary cardiovascular risk reduction therapy (consisting of an aspirin, a $\beta$-blocker, an ACE inhibitor or an angiotensin II receptor blocker and a statin) is needed for all patients with ACS. Less than $80 \%$ of patients with ACS in Qatar use this combination after discharge. This study is aimed to evaluate the effectiveness of clinical pharmacistdelivered intervention at discharge and tailored followup postdischarge on decreasing hospital readmissions, emergency department (ED) visits and mortality among patients with ACS.

Methods and analysis: A prospective, randomised controlled trial will be conducted at the Heart Hospital in Qatar. Patients are eligible for enrolment if they are at least 18 years of age and are discharged from any non-surgical cardiology service with ACS. Participants will be randomised into 1 of 3 arms: (1) 'control' arm which includes patients discharged during weekends or after hours; (2) 'clinical pharmacist delivered usual care at discharge' arm which includes patients receiving the usual care at discharge by clinical pharmacists; and (3) 'clinical pharmacist-delivered structured intervention at discharge and tailored follow-up postdischarge' arm which includes patients receiving intensive structured discharge interventions in addition to 2 follow-up sessions by intervention clinical pharmacists. Outcomes will be measured by blinded research assistants at 3, 6 and 12 months after discharge and will include: all-cause hospitalisations and cardiac-related hospital readmissions (primary outcome), all-cause mortality including cardiac-related mortality, ED visits including cardiac-related ED visits, adherence to medications and treatment burden. Percentage of readmissions between the 3 arms will be compared on intent-totreat basis using $\chi^{2}$ test with Bonferroni's adjusted pairwise comparisons if needed.

Ethics and dissemination: The study was ethically approved by the Qatar University and the Hamad Medical Corporation Institutional Review Boards.

\section{Strengths and limitations of this study}

- This is the first randomised controlled study that investigates the impact of clinical pharmacists as direct patient care team members at discharge and postdischarge on patients with acute coronary syndromes in Qatar and probably in the Middle East.

- The study results will show the extent to which a pharmacist-delivered pharmaceutical care intervention is effective and feasible in the cardiovascular setting.

- The study will help in setting and integrating an effective standard of care for discharge and follow-up procedures for cardiac patients and in improving the management of one of the most prevalent chronic diseases in Qatar.

- The study limitation is that study results may not be generalisable to other countries.

The results shall be disseminated in international conferences and peer-reviewed publications.

Trials registration number: NCT02648243; pre-results.

\section{INTRODUCTION}

Cardiovascular diseases (CVDs) are considered a leading cause of death, with an estimated 17.5 million deaths worldwide in 2012. Coronary heart diseases including acute coronary syndrome (ACS) account for $31 \%$ of all deaths. ${ }^{1}$ Patients with ACS have an increased risk of future recurrence of cardiovascular and non-coronary atherosclerotic events. ${ }^{2}$ Consequently, all patients post-ACS should be prescribed secondary cardiovascular risk reduction therapy also known as 'secondary prevention'. Unless contraindicated, 
this therapy should be started in all patients with ACS before hospital discharge. ${ }^{4}$ Internationally recognised clinical practice guidelines by the American College of Cardiology (ACC)/American Heart Association (AHA), European Society of Cardiology (ESC), and the National Institute for Health and Care Excellence (NICE), strongly recommend optimisation of secondary prevention drug therapies following ACS. ${ }^{5-9}$ According to the ACC/AHA guidelines, all patients with ACS should receive indefinite treatment with aspirin, a $\beta$-blocker, an ACE inhibitor (ACEI) or alternatively an angiotensin II receptor blocker (ARB), and a statin. In addition, a platelet P2Y12 receptor blocker (clopidogrel or prasugrel or ticagrelor) may be prescribed. ${ }^{5} 61011$ These evidence-based recommendations are predicated on many studies that have demonstrated the benefits of using the quadruple combination of secondary prevention medications (antiplatelet, statin, $\beta$-blocker, and ACEI or ARB) at discharge. ${ }^{12} 13$ Nevertheless, there is a corresponding documented evidence of underusage and of low adherence to secondary prevention medications among patients with ACS in many countries including the USA, Canada and Qatar. ${ }^{14-19}$ Non-adherence to and early discontinuation of ACS secondary prevention medications are associated with an increased risk of subsequent adverse cardiovascular events, hospital readmissions and mortality. ${ }^{20-26}$

The phase after hospital discharge is a vulnerable period and a critical one for patients with ACS. ${ }^{27}$ Often, patients are left unprepared at discharge and many do not receive sufficient education about their discharge medications or treatment plan. ${ }^{28}$ Evidence from the literature supports that restructuring the patient discharge process to include activities such as discharge medication reconciliation and counselling, and postdischarge monitoring and follow-up can decrease the frequency of these adverse outcomes. ${ }^{29-33}$

Clinical pharmacists are well positioned to provide pharmaceutical care interventions as patients transition between different healthcare settings. Their role has been well defined by many professional organisations including the International Pharmaceutical Federation (FIP), the WHO, the European Society of Clinical Pharmacy (ESCP), and the American College of Clinical Pharmacy (ACCP). ${ }^{34-36}$ For example, ESCP defines the role of clinical pharmacists as that which promotes the safe and appropriate use of medications with the aim of achieving the desired therapeutic outcomes. ${ }^{36}$ In addition, many studies have demonstrated the impact of pharmacist interventions which include patient education at discharge and/or follow-up in decreasing the rate of preventable adverse drug events, hospital readmissions and emergency department (ED) visits. ${ }^{31} 3237-39$ Undoubtedly, the incorporation of pharmacists into the CVD healthcare team can improve the usage of CVD medications ${ }^{40}$ and increase patient adherence to drug therapy. This can also potentially decrease the number of hospitalisations, ED visits and mortality. ${ }^{41}{ }^{42}$ The role of pharmacists in CVD management is well recognised by professional pharmacy bodies. ${ }^{34-36}$ In particular, the ACCP and the Institute for Healthcare Improvement have developed a best practices model for pharmacist-based discharge counselling for patients with heart failure and myocardial infarction which targets, but not limited to, medication use, barriers for adherence and the patient's role in self-care. ${ }^{43}$

According to the WHO 2014 report, CVDs accounted for $24 \%$ of all deaths in Qatar. ${ }^{44}$ Moreover, the annual report of Hamad Medical Corporation (HMC) in 2009 indicated that the highest number of reported deaths in Qatar was attributed to diseases of the circulatory system. The medication taking behaviour of the quadruple evidence-based secondary prevention medications was previously assessed in a cohort of 31 ACS outpatients at the Heart Hospital (HH). The self-reported continuation rates for secondary prevention medications 6 months after the last hospital discharge decreased from $100 \%$ to $77 \%$ for $\beta$-blockers, $90 \%$ to $79 \%$ for ACE inhibitors, $100 \%$ to $97 \%$ for statins and $84 \%$ to $52 \%$ for dual antiplatelet therapy. The most common reasons for medication discontinuation were patients' experiences of intolerable medication adverse effects, high number of prescribed medications at the time of discharge and the fear of experiencing a medication adverse effect. ${ }^{19}$ This creates a significant opportunity for the clinical pharmacists to improve CVD management and outcomes in Qatar.

\section{RATIONALE}

To the best of our knowledge, this study is the first in Qatar to assess the effectiveness of a clinical pharmacistdelivered intervention among patients with ACS on cardiovascular-related outcomes. If proven to be effective, this intervention will potentially be adopted in Qatar and other cardiology centres in the region to ultimately improve cardiac care and outcomes in patients with ACS and to decrease ACS-related healthcare resource usage. Furthermore, the study will act as a catalyst to develop standards for discharge and follow-up programmes for patients with coronary heart disease in Qatar and other developing nations.

\section{HYPOTHESIS}

We hypothesise that an intensive structured clinical pharmacist-delivered intervention consisting of medication reconciliation and counselling at discharge and tailored follow-up postdischarge will decrease hospital readmissions, ED visits and all-cause mortality among patients with ACS at 3,6 and 12 months after hospital discharge when compared with 'no-pharmacist intervention' or 'usual pharmacist care'. We also hypothesise that the effect of the intensive structured pharmacist intervention will increase patients' adherence to evidence-based secondary prevention medications for recurrent cardiovascular events and will decrease treatment burden as perceived by the patients. 


\section{OBJECTIVES}

This protocol describes a study that is currently being implemented at $\mathrm{HH}$, one of the tertiary care hospitals under the HMC in Qatar.

The study is primarily aimed to evaluate the effectiveness of clinical pharmacist-delivered intervention at discharge and tailored follow-up postdischarge on decreasing hospital readmissions.

The secondary objectives of the study are to evaluate the effectiveness of the intervention on: (1) decreasing ED visits, and mortality among patients with ACS; (2) improving patient adherence to evidence-based secondary prevention therapy; and (3) decreasing treatment burden as perceived by patients with ACS.

\section{METHODS}

\section{Study design}

The study is a prospective randomised controlled trial assessing the effectiveness of a structured intensive clinical pharmacist-delivered intervention consisting of medication reconciliation and counselling at discharge and tailored follow-up postdischarge on: (1) hospital readmissions including cardiac-related hospital readmissions; (2) ED visits; (3) all-cause mortality including cardiac-related mortality; (4) adherence to evidencebased secondary prevention medications and (5) selfreported treatment burden.

\section{Study arms}

The study will have three arms: (1) 'control' arm (control arm) which includes patients discharged during weekends or after the clinical pharmacists' working hours; (2) 'clinical pharmacist delivered usual care at discharge' arm (usual care arm) which includes patients receiving the usual care at discharge by the clinical pharmacists and (3) 'clinical pharmacist delivered intensive structured intervention at discharge and tailored follow-up postdischarge' arm (intervention arm) which includes patients receiving a structured discharge intervention in addition to two follow-up sessions (around $30 \mathrm{~min}$ each) at $\sim 4$ and 8 weeks of discharge. The usual care delivered by cardiologists is similar across all the study arms and no modification of level of care was required by the present study.

\section{Study site}

The study is being implemented at $\mathrm{HH}$, which is one of the new hospitals under the umbrella of the HMC. The centre is equipped with a 20-bed coronary care unit, a 12-bed cardiothoracic intensive care unit, a 24-bed surgical high dependency unit and 60 ward beds with continuous telemetry monitoring rooms. It provides inpatient and outpatient tertiary care services for more than $95 \%$ of the patients with ACS in Qatar. ${ }^{45}$ Around 3000 patients with ACS are admitted to the HH annually. These patients receive their secondary prevention medications from the hospital's outpatient pharmacy with a nominal charge of about 150 QR (Qatar Riyal) per prescription refill.

\section{Eligibility criteria}

Patients are eligible for enrolment into the study if they are at least 18 years of age and are admitted to and discharged from any non-surgical cardiology service at $\mathrm{HH}$ with a diagnosis of ACS regardless of whether it is their first ACS attack or not. Patients will be excluded from the study if any of the following criteria is met: severe visual impairment, severe hearing impairment, inability to communicate in either English or Arabic, mental or psychiatric illness, delirium or severe dementia, cognitive impairment, incomprehensible speech, planned discharge to a location other than home (eg, long-term care facility, nursing home, other medicine units, etc), plan for coronary artery bypass graft (CABG) surgery during hospitalisation, plan to leave Qatar in the next 12 months, and a terminal illness with a high likelihood of death in the next 12 months.

\section{Screening and recruitment of patients}

Trained research assistants (RAs) will identify potential participants through reviewing the HH's medical records according to the study's inclusion and exclusion criteria. The RAs will approach identified participants, provide them with information about the study and obtain written informed consent, including permission to access the patient's medical records.

After obtaining the informed consent, the RAs will document through medical chart review and patient interview the patient's baseline characteristics including demographics and comorbidities such as age, gender, country of origin, type of ACS, education level, medical conditions (eg, diabetes mellitus, heart failure, hyperlipidaemia, hypertension, renal dysfunction, obesity, depression, chronic obstructive pulmonary disease, cerebrovascular accident, etc), past and current smoking status, current prescription and over-the-counter medications, clinical parameters (eg, most recent blood pressure, pulse rate, lipid profile, blood glucose, weight, height), and inpatient treatment modality (eg, medical management vs CABG vs percutaneous coronary intervention).

\section{Randomisation}

At $\mathrm{HH}$ on arrival to the ED, patients with ACS are usually allocated to different treatment teams by the triage nurse based on the capacity of the teams. These treatment teams are denoted by the letters $\mathrm{A}, \mathrm{B}, \mathrm{C}$ and $\mathrm{D}$; typically, each team has a clinical pharmacist as a member. This inherent process of randomly allocating patients to treatment teams serves as the basis of randomisation in this study. First, consenting patients who happen to be allocated to the teams that have the intervention clinical pharmacists will be naturally considered in the intervention arm. Second, consenting patients who happen to be allocated to the teams that have the 
non-intervention clinical pharmacists will be considered in the usual care arm. Finally, consenting patients who are discharged on weekends and after the clinical pharmacists' working hours will be naturally randomised into the control arm.

Assigning patients to the different teams is the usual practice at $\mathrm{HH}$. Allocation or selection bias is unlikely to happen in the study due to the fact that the clinical pharmacists have no role in patients' allocation.

\section{Description of the intervention}

1. 'Control' arm: in this arm, patients will typically receive routine discharge instructions and medication information by nurses and/or treating physicians during hospital discharge. This arm comprises of consenting patients who are discharged during weekends and/or at times outside the clinical pharmacists' working hours. These patients will not have any contact with the clinical pharmacists at discharge.

2. 'Clinical pharmacist delivered usual care at discharge' arm: in this arm, the clinical pharmacist will provide the usual care to patients at discharge which includes:

- Reviewing and reconciling patient's discharge medications.

- Documenting and resolving any identified discrepancies and drug-related problems as appropriate.

- Providing the usual general discharge counselling.

3. 'Clinical pharmacist-delivered intensive structured intervention at discharge and tailored follow-up postdischarge' arm: in this arm, the clinical pharmacist will deliver a structured discharge intervention in addition to two coordinated follow-up sessions (around $30 \mathrm{~min}$ each session) at 4 and 8 weeks postdischarge. Specifically, the intervention clinical pharmacist will:

- Provide structured counselling to the patient by: reviewing the patient's baseline knowledge and experience; educating them about coronary artery disease (CAD) and about the goals of therapy; reviewing important information pertinent to each medication (instructions of use, anticipated benefits, common side effects, appropriate actions to take in case side effects occur, etc); providing additional counselling and monitoring for medications requiring exceptional instructions if applicable (eg, warfarin); emphasising the differences between the patient's preadmission and discharge medication regimens; providing recommendations for alternatives in case of contraindications to any of the secondary prevention medications as per evidence-based guidelines; discussing the drugs and herbs that the patient should generally avoid; discussing risk factor control (eg, diabetes, hypertension, dyslipidaemia, etc); general lifestyle modification counselling including weight loss, appropriate physical activity, dietary modification, smoking cessation, etc; educating the patient on the importance of adherence to their medications; assessing the patient's potential barriers for medication adherence and for adopting lifestyle modifications (eg, literacy, language barrier, cost, polypharmacy, etc); and reviewing with the patient some strategies to improve medication adherence (eg, how to prevent or alleviate side effects, etc); assessing and addressing any patient's concerns; and if needed, asking the patient to 'teach-back' key features of their medication regimens to confirm comprehension of important medical and medication instructions.

- Ensure that a follow-up plan for medication monitoring after discharge is communicated to the patient.

- Provide the patient with a personalised medication timetable that lists indication, time and directions of use, in addition to the relative common side effects of each medication.

- Offer the patient a pill box and teach him/her how to fill it using the medication timetable as a guide.

- Give the patient information leaflets that are developed by the study team. Each patient information leaflet will be related to a specific medication or class of medications and will contain relevant medication information (eg, indication, directions for use, warnings, side effects, benefits, what to do in case of missed dose, storage recommendations, etc) in a lay language (English or Arabic) that is easily readable and comprehensible by the patient. The leaflets will be adapted from reputable drug information resources such as UptoDate and Micromedex. ${ }^{46} 47$

- Schedule two follow-up sessions with the patients at 4 and 8 weeks postdischarge. These follow-up sessions will be delivered over the phone. During these sessions, the intervention pharmacists will: review the medications with the patient to identify any discrepancies between his/her current medications and his/her discharge medication list; identify any drug-related problems (eg, side effects, etc); confirm that the medications are filled. In case any of the medications is not filled or stopped, the pharmacist will: elicit the reason from the patient or contact the patient's physician as necessary; confirm that the patient understands each medication's instructions and any gaps in understanding will be determined and addressed; assess the patient's lifestyle (eg, diet, etc) and provide counselling as needed; assess the patient's adherence to medications and elicit reasons for non-adherence if applicable (eg, complicated regimens, cost, medication intolerable side effects, difficulty in remembering to take the medications, etc); re-emphasise the importance of adherence to secondary prevention medications in order to reduce the risk of recurrent cardiac events; in case 
of any identified non-adherence, the pharmacist will work with the patient to promote adherence by offering strategies such as simplifying medication regimens (eg, sustained release, etc), tailoring medication regimens according to the patient's daily routine (eg, identifying a daily activity that the patient regularly performs at about the time he/she should take his/her medications and explain to the patient to take medications at this time, encouraging the patient to use an alarm to remember the next dose), managing side effects, offering adherence aids, etc; and document the patient education during follow-up sessions on a newly created 'patient follow-up' form as well as in the patient's medical record.

\section{Intervention clinical pharmacists}

Three clinical pharmacists from $\mathrm{HH}$ are participating in the 'intervention arm' of the study. The selection of pharmacists in this arm was based on pharmacist availability during the study period, their willingness to participate, their credentials and their workload. Intervention pharmacists should hold a Doctor of Pharmacy (PharmD) degree and should have completed at least 1 year of residency programme or at least 2 years of pharmacy experience.

The study team provided the intervention pharmacists with a 3-hour orientation session. The session covered a refresher on the following: the current guidelines on the secondary prevention of ACS, non-pharmacological management, pharmaceutical care's basic concepts, communication and counselling skills, medication reconciliation, medication adherence assessment and strategies to improve medication adherence. The orientation also covered the study protocol and the study forms that the pharmacists would need to fill. In addition, the pharmacists were orientated to provide the intervention in a standardised fashion, while tailoring it to the patient's needs.

\section{Study piloting}

Prior to the beginning of the study, the intervention clinical pharmacists will pilot the feasibility of the study in the presence of the study team. Piloting will occur by delivering the intervention to two patients per pharmacist. With the patient's permission, a sample of the counselling will be audio-recorded and the pharmacists will be provided with individual feedback. Necessary refinements will be made to all the study logistics and study forms based on the piloting experience.

\section{Ethical consideration and dissemination}

All participants' folders will be stored in a passwordprotected database on the site laptop. Only the study personnel can access these folders. In addition, all data retained in the password-protected database will be maintained along with all related study documentation in a locked cabinet at Qatar University (QU) College of Pharmacy.
The results of this study will be presented in local, regional and international conferences as well as published in international peer-reviewed journals.

\section{Outcome measures}

All surviving patients in the three study arms will meet the study RAs who will be blinded to treatment group at $\sim 3,6$ and 12 months after hospital discharge. The outcome measures include:

- Primary outcome: all-cause hospitalisations and cardiac-related hospital readmissions including hospitalisations for cardiac events, exacerbation of heart failure or arrhythmia. This outcome will be assessed by checking HH and HMC medical records.

- Secondary outcome: ED visits including cardiac-related ED visits. This will be assessed by checking $\mathrm{HH}$ and HMC medical records. Any ED visit in which a patient is subsequently hospitalised will be counted as a hospital readmission.

- Secondary outcome: all-cause mortality including cardiac-related mortality: this outcome will be assessed by checking $\mathrm{HH}$ and HMC medical records during the designated follow-up periods.

- Secondary outcome: patient adherence to evidencebased secondary prevention medications for CAD will be measured using three strategies:

1. Patients' self-report of taking all and each individual prescribed secondary prevention evidence-based medications for CAD. This will be assessed by asking the patient to state which medications he/she is currently taking and then compare this list to the patient's discharge medication list. Any discrepancies (eg, change in or missing medication dose or frequency, or route of administration or omissions, additions substitutions or duplications) will be noted and causes for these discrepancies will be discussed with the patient to determine if they were intentional or not. Medication changes will be considered intentional if they were made by the patient's physician. If the patient in any study arm reports that a medication is discontinued by his/her physician, the physician will be contacted by phone or email to confirm and obtain the reason for the discontinuation. Patient will be considered adherent if the physician verified discontinuing the medication.

2. Administration of the Adherence to Refills and Medications Scale (ARMS). ${ }^{48}$

3. Patients' adherence to all and to each individual prescribed secondary prevention medications for CAD as per the prescription refill records at the $\mathrm{HH}$ outpatient pharmacy covering the period from the patient hospital discharge until the day of outcome assessment. The patient will be considered adherent by pharmacy prescription records if the proportion of days covered (PDC) for each of the secondary prevention medications ( $\beta$-blocker, aspirin, statin, ACEI or ARB if applicable) from discharge is larger than $75 \%$. A PDC value of more than $75 \%$ is selected 
based on previous studies that assessed adherence in postmyocardial infarction patients. ${ }^{49}$ If one of the medications is not prescribed at discharge, the PDC to only the prescribed medications will be used to determine the overall adherence. To be classified as having overall adherence, the patient must have a $\mathrm{PDC} \geq 75 \%$ for each medication. For example, if the patient's PDC for one of the medications is $80 \%$ but PDC for another medication is $50 \%$, the patient would be classified as non-adherent. A patient will be considered non-adherent in case he/she is found non-adherent in any modality even if the same patient is found adherent by the other two modalities.

- Treatment burden: will be assessed using the culturally adapted and translated Treatment Burden Questionnaire (TBQ). ${ }^{50} 51$

\section{Participant flow through the study}

For more information regarding the flow of participants during the study, please see figure 1 .

\section{Data analysis and sample size calculation}

The trial will be analysed according to the CONSORT guidelines. Baseline demographic and clinical characteristics between the three study arms will be compared using one-way analysis of variance (ANOVA) for the numeric variables (such as age) and the $\chi^{2}$ test for categorical variables (such as gender). Alternative non-parametric tests will be used if needed. The primary analysis will involve comparing the main outcome of percentage of readmissions between the three study arms at 6 months using the $\chi^{2}$ test. Pairwise comparisons using Bonferroni's adjustment for the significance level will be used if needed. Logistic regression will be used to
Figure 1 Participants flow during the study.

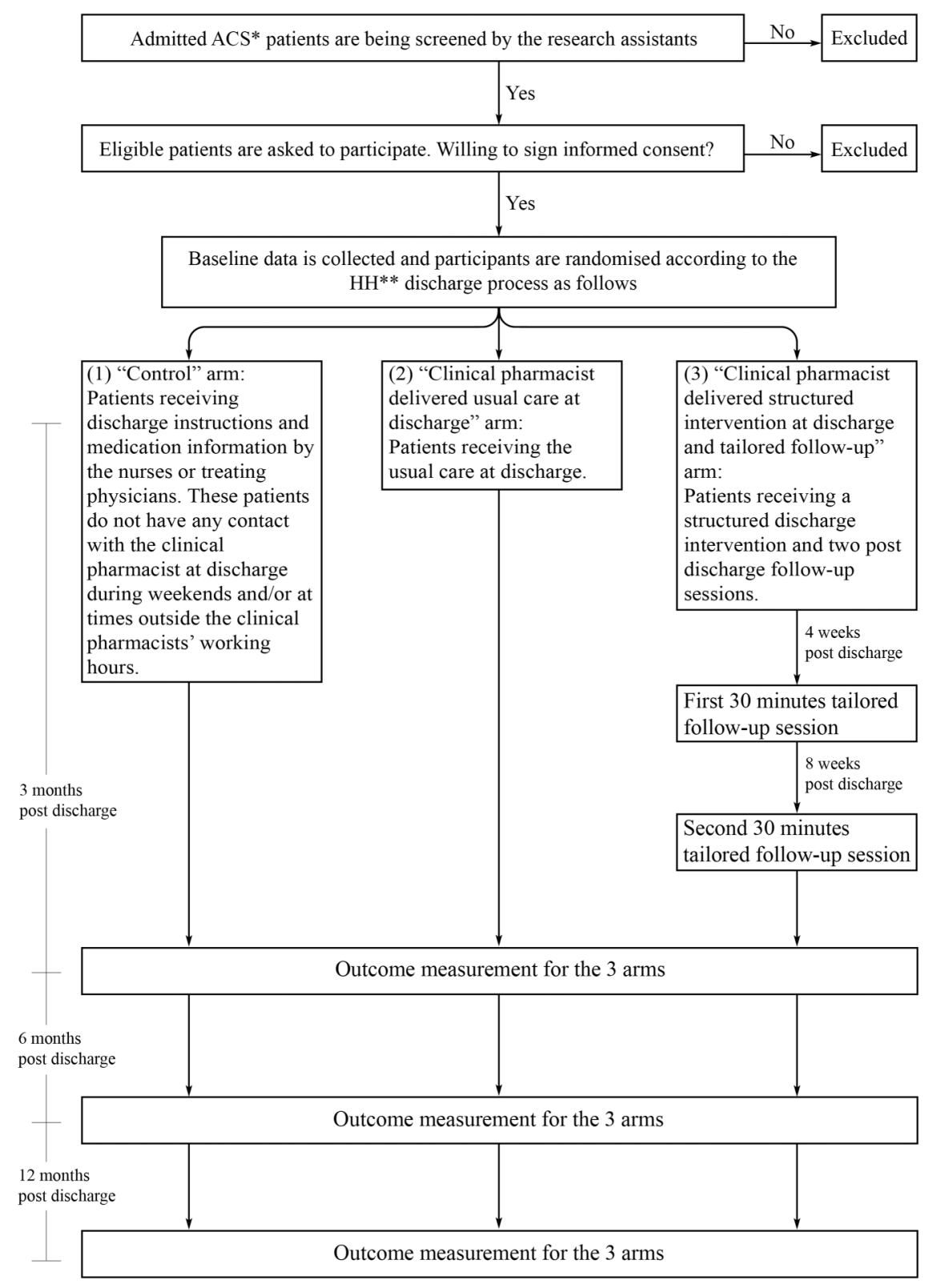


compute the unadjusted OR between the three groups (with the control arm being the reference group) along with $95 \%$ CIs. The number needed to treat (NNT) will be computed when significance is achieved in the main outcome between any two pairs of arms. This analysis will be carried out for the two time periods 3 and 12 months. Secondary analysis will involve obtained adjusted OR for the primary outcome where adjustment is done for any imbalances deemed clinically important in demographic and baseline clinical variables between the study arms. In addition, the primary outcome will be adjusted for the possible clustering effect within each team using mixed effects regression analysis. Also the secondary outcomes of all-cause mortality and adherence will be analysed in a similar manner.

The treatment burden will be compared between the study arms using the $\chi^{2}$ test followed by multiple logistic regressions or one-way ANOVA followed by multiple linear regressions. Intent-to-treat principal will be used for the analysis. Two sensitivity analyses will be performed one using multiple imputations by chained equations for the missing data and the other using per protocol methodology. Also the effect of adherence on the primary analysis will be assessed by adding this variable to the multiple logistic regression models. Statistical analyses will be conducted using the Statistical Package of Social Sciences (IBM SPSS) V.22. In all analyses, the level of significance will be set at $5 \%$. The sample size is based on the primary analysis of comparing the main outcome between the three study arms. Assuming that the 6-month readmission among the patients on the control arm is about $20 \%$ with 125 patients per arm, we will be able to detect an effect size of 0.025 between the three groups corresponding to an absolute decrease of $10 \%$ between the intervention arm and usual care arm and $9 \%$ between this latter group and the control arm with a significance level of $5 \%$ and a power of $80 \% .^{51}$ Approximately 250-300 patients are admitted to $\mathrm{HH}$ per month with ACS of which 10-15\% are discharged on weekends or after the clinical pharmacists' working hours. On average, length of stay of patients with ACS ranges from 72 to 96 hours. The recruitment phase of the study is over 12 months. Assuming that the RAs recruit 3 participants in each arm every week, the estimated sample size of 125 patients per arm would be achieved in 42 weeks.

\section{Limitations}

The study is not without limitations. True randomisation of participants is not possible. Participants are allocated to the three arms by the triage nurse based on the capacity of the different teams. This is the most feasible and natural way of randomisation given the nature of practice in the study setting. Furthermore, the study involves only one hospital in Qatar; therefore, the study results may not be generalisable to other centres in the country. Nevertheless, $\mathrm{HH}$ is the main centre that offers cardiac services to Qatar's population which makes this limitation less concerning.
Contributors AZ was involved in drafting the protocol and is responsible for the treatment burden part of the project. AA has made substantial contributions to the conception of the research idea, design of the study, and was involved in drafting and critically revising this protocol for important intellectual content. NK contributed in major way in discussions related to the design of the research project, developing and adapting the tools related to treatment burden, and review of all the drafts throughout the project including the protocol. ZM assisted in writing the data analysis and sample size calculation of the protocol. RK had input in reviewing the protocol and is making substantial contribution in executing the study at $\mathrm{HH}$. SA had input in reviewing the protocol and is making substantial contribution in executing the study at $\mathrm{HH}$. MH is the lead principal investigator of the study leading its design and execution. She wrote the study protocol and manuscript.

Funding The work was supported by Qatar University Internal grant number QUUG-CPH-CPH-14/15-2.

Competing interests None declared.

Patient consent Obtained.

Ethics approval The study protocol, informed consent forms, recruitment procedures, data collection forms and all other relevant documents were reviewed and approved by the Qatar University (QU) and the Hamad Medical Corporation (HMC) Institutional Review Boards. Approval numbers are QU-IRB 527-EA/15 and 15319/15, respectively.

Provenance and peer review Not commissioned; externally peer reviewed.

Open Access This is an Open Access article distributed in accordance with the Creative Commons Attribution Non Commercial (CC BY-NC 4.0) license, which permits others to distribute, remix, adapt, build upon this work noncommercially, and license their derivative works on different terms, provided the original work is properly cited and the use is non-commercial. See: http:// creativecommons.org/licenses/by-nc/4.0/

\section{REFERENCES}

1. WHO. Cardiovascular diseases. Secondary cardiovascular diseases 2013. http://www.who.int/mediacentre/factsheets/fs317/en/index.html (accessed 14 Oct 2014).

2. Hiratzka LF, Eagle KA, Liang L, et al, Get With the Guidelines Steering Committee. Atherosclerosis secondary prevention performance measures after coronary bypass graft surgery compared with percutaneous catheter intervention and nonintervention patients in the Get With the Guidelines database. Circulation 2007;116(11 Suppl):I207-I12.

3. Witt BJ, Brown RD, Jacobsen SJ, et al. A community-based study of stroke incidence after myocardial infarction. Ann Intern Med 2005;143:785-92.

4. DiPiro JT, Robert L, Yee GC, et al. Pharmacotherapy: a pathophysiologic approach. McGraw-Hill Education, 2014

5. Smith SC, Allen J, Blair SN, et al. AHA/ACC guidelines for secondary prevention for patients with coronary and other atherosclerotic vascular disease: 2006 update: endorsed by the National Heart, Lung, and Blood Institute. J Am Coll Cardiol 2006;47:2130-9.

6. Smith SC, Benjamin EJ, Bonow RO, et al. AHA/ACCF secondary prevention and risk reduction therapy for patients with coronary and other atherosclerotic vascular disease: 2011 update: a guideline from the American Heart Association and American College of Cardiology Foundation endorsed by the World Heart Federation and the Preventive Cardiovascular Nurses Association. J Am Coll Cardiol 2011;58:2432-46.

7. Bassand JP, Hamm CW, Ardissino D, et al. Guidelines for the diagnosis and treatment of non-ST-segment elevation acute coronary syndromes. Eur Heart J 2007;28:1598-660.

8. Van de Werf F, Bax J, Betriu A, et al. Management of acute myocardial infarction in patients presenting with persistent ST-segment elevation. Eur Heart J 2008;29:2909-45

9. Jones K, Saxon L, Cunningham W, et al. Secondary prevention for patients after a myocardial infarction: summary of updated NICE guidance. BMJ 2013;347:f6544.

10. Anderson JL, Adams CD, Antman EM, et al. ACC/AHA 2007 guidelines for the management of patients with unstable angina/ non-ST-elevation myocardial infarction: a report of the American College of Cardiology/American Heart Association Task Force on Practice Guidelines (Writing Committee to revise the 2002 guidelines 
for the management of patients with unstable angina/ non-ST-elevation myocardial infarction) developed in collaboration with the American College of Emergency Physicians, the Society for Cardiovascular Angiography and Interventions, and the Society of Thoracic Surgeons endorsed by the American Association of Cardiovascular and Pulmonary Rehabilitation and the Society for Academic Emergency Medicine. J Am Coll Cardiol 2007;50: e1-e157.

11. Kushner FG, Hand M, Smith SC, et al. 2009 focused updates: ACC/ AHA guidelines for the management of patients with ST-elevation myocardial infarction (updating the 2004 guideline and 2007 focused update) and ACC/AHA/SCAI guidelines on percutaneous coronary intervention (updating the 2005 guideline and 2007 focused update) a report of the American College of Cardiology Foundation/American Heart Association Task Force on Practice Guidelines. J Am Coll Cardiol 2009;54:2205-4112.

12. Mukherjee D, Fang J, Chetcuti S, et al. Impact of combination evidence-based medical therapy on mortality in patients with acute coronary syndromes. Circulation 2004;109:745-9.

13. Expert Panel on Detection, Evaluation, and Treatment of High Blood Cholesterol in Adults. Executive summary of the third report of the National Cholesterol Education Program (NCEP) Expert Panel on detection, evaluation, and treatment of high blood cholesterol in adults (Adult Treatment Panel III). JAMA 2001;285:2486-97.

14. Lewis SJ, Robinson JG, Fox KM, et al. Underutilisation of cardiovascular medications among at-risk individuals. Int J Clin Pract 2010;64:604-10.

15. Kumbhani DJ, Fonarow GC, Cannon CP, et al. Predictors of adherence to performance measures in patients with acute myocardial infarction. Am J Med 2013;126:74.e1-9.

16. Yan AT, Yan RT, Tan M, et al. Optimal medical therapy at discharge in patients with acute coronary syndromes: temporal changes, characteristics, and 1-year outcome. Am Heart J 2007;154:1108-15.

17. Naderi SH, Bestwick JP, Wald DS. Adherence to drugs that prevent cardiovascular disease: meta-analysis on 376,162 patients. Am J Med 2012;125:882-7.e1.

18. Bi Y, Gao R, Patel A, et al. Evidence-based medication use among Chinese patients with acute coronary syndromes at the time of hospital discharge and 1 year after hospitalization: results from the Clinical Pathways for Acute Coronary Syndromes in China (CPACS) study. Am Heart J 2009;157:509-16.e1.

19. Abu Samaha RAS, Mohammed S, El Hajj M. Assessment of patient adherence to secondary preventive medications post acute coronary syndrome. Qatar. (Poster). Doha, Qatar: College of Pharmacy 4th Annual Research Forum. Qatar University, 2014.

20. Wei L, Wang J, Thompson P, et al. Adherence to statin treatment and readmission of patients after myocardial infarction: a six year follow up study. Heart 2002;88:229-33.

21. Wei L, Flynn R, Murray GD, et al. Use and adherence to beta-blockers for secondary prevention of myocardial infarction: who is not getting the treatment? Pharmacoepidemiol Drug Saf 2004:13:761-6.

22. Ho PM, Spertus JA, Masoudi FA, et al. Impact of medication therapy discontinuation on mortality after myocardial infarction. Arch Intern Med 2006;166:1842-7.

23. Spertus JA, Kettelkamp R, Vance C, et al. Prevalence, predictors, and outcomes of premature discontinuation of thienopyridine therapy after drug-eluting stent placement results from the PREMIER registry. Circulation 2006;113:2803-9.

24. Rublee DA, Chen SY, Mardekian J, et al. Evaluation of cardiovascular morbidity associated with adherence to atorvastatin therapy. Am J Ther 2012;19:24-32.

25. Gehi AK, Ali S, Na B, et al. Self-reported medication adherence and cardiovascular events in patients with stable coronary heart disease: the heart and soul study. Arch Intern Med 2007;167:1798-803.

26. Kumbhani DJ, Steg PG, Cannon CP, et al. Adherence to secondary prevention medications and four-year outcomes in outpatients with atherosclerosis. Am J Med 2013;126:693-700.e1.

27. Coleman EA, Berenson RA. Lost in transition: challenges and opportunities for improving the quality of transitional care. Ann Intern Med 2004;141:533-6.

28. Makaryus AN, Friedman EA. Patients' understanding of their treatment plans and diagnosis at discharge. Mayo Clin Proc 2005;80:991-4.
29. Forster AJ, Murff HJ, Peterson JF, et al. The incidence and severity of adverse events affecting patients after discharge from the hospital. Ann Intern Med 2003;138:161-7.

30. Halasyamani L, Kripalani S, Coleman E, et al. Transition of care for hospitalized elderly patients-development of a discharge checklist for hospitalists. J Hosp Med 2006;1:354-60.

31. Dudas V, Bookwalter T, Kerr KM, et al. The impact of follow-up telephone calls to patients after hospitalization. Am J Med 2001;111:26-30.

32. Boockvar KS, Carlson LaCorte H, Giambanco V, et al. Medication reconciliation for reducing drug-discrepancy adverse events. Am J Geriatr Pharmacother 2006;4:236-43.

33. Lee JK, Grace KA, Taylor AJ. Effect of a pharmacy care program on medication adherence and persistence, blood pressure, and low-density lipoprotein cholesterol: a randomized controlled trial. JAMA 2006;296:2563-71.

34. Developing pharmacy practice: a focus on patient care. Secondary developing pharmacy practice: a focus on patient care 2006. http:// www.who.int/medicines/publications/WHO_PSM_PAR_2006.5.pdf (accessed 14 Mar 2016).

35. Definition of Clinical Pharmacy. http://www.accp.com/stunet compass/definition.aspx (accessed 14 Mar 2016).

36. What is clinical pharmacy? http://www.escpweb.org/ (accessed 14 Mar 2016).

37. Schnipper JL, Kirwin JL, Cotugno MC, et al. Role of pharmacist counseling in preventing adverse drug events after hospitalization. Arch Intern Med 2006;166:565-71.

38. Crotty M, Rowett D, Spurling $L$, et al. Does the addition of a pharmacist transition coordinator improve evidence-based medication management and health outcomes in older adults moving from the hospital to a long-term care facility? Results of a randomized, controlled trial. Am J Geriatr Pharmacother 2004;2:257-64

39. Jack BW, Chetty VK, Anthony D, et al. A reengineered hospital discharge program to decrease rehospitalization: a randomized trial. Ann Intern Med 2009;150:178-87.

40. Bailey TC, Noirot LA, Blickensderfer A, et al. An intervention to improve secondary prevention of coronary heart disease. Arch Intern Med 2007;167:586-90.

41. Ponniah A, Anderson B, Shakib S, et al. Pharmacists' role in the post-discharge management of patients with heart failure: a literature review. J Clin Pharm Ther 2007;32:343-52.

42. Blenkinsopp A, Anderson C, Armstrong M. Systematic review of the effectiveness of community pharmacy-based interventions to reduce risk behaviours and risk factors for coronary heart disease. J Public Health 2003;25:144-53.

43. Wiggins BS, Rodgers JE, DiDomenico RJ, et al. Discharge counseling for patients with heart failure or myocardial infarction: a best practices model developed by members of the American College of Clinical Pharmacy's Cardiology Practice and Research Network based on the Hospital to Home $(\mathrm{H} 2 \mathrm{H})$ Initiative. Pharmacotherapy 2013;33:558-80.

44. NCD country profile: Qatar 2014. http://www.who.int/nmh/countries/ en/\#Q (accessed 31 Mar 2016).

45. El-Menyar A, Ahmed E, Albinali $\mathrm{H}$, et al. Mortality trends in women and men presenting with acute coronary syndrome: insights from a 20-year registry. PLOS ONE 2013;8:e70066

46. Micromedex® Solutions, 2016. https://www.micromedexsolutions. com/home/dispatch/ssl/true (accessed 31 Mar 2016)

47. UpToDate, 2016. http://www.uptodate.com/ (accessed 31 Mar 2016)

48. Kripalani S, Risser J, Gatti ME, et al. Development and evaluation of the Adherence to Refills and Medications Scale (ARMS) among low-literacy patients with chronic disease. Value Health 2009;12:118-23.

49. Horwitz RI, Viscoli CM, Donaldson R, et al. Treatment adherence and risk of death after a myocardial infarction. Lancet 1990;336:542-5.

50. Tran VT, Harrington M, Montori VM, et al. Adaptation and validation of the Treatment Burden Questionnaire (TBQ) in English using an internet platform. BMC Med 2014;12:109.

51. Sangu PV, Ranasinghe I, Aliprandi Costa B, et al. Trends and predictors of rehospitalisation following an acute coronary syndrome: report from the Australian and New Zealand population of the Global Registry of Acute Coronary Events (GRACE). Heart 2012:98:1728-31. 